\title{
Therapeutic Arteriogenesis/Angiogenesis for Peripheral Arterial Disease by Nanoparticle-Mediated Delivery of Pitavastatin into Vascular Endothelial Cells
}

\author{
Takuya Matsumoto, MD, PhD, FACS, ${ }^{1,2}$ Sho Yamashita, MD, ${ }^{1,2}$ Shinichiro Yoshino, MD, ${ }^{2}$ \\ Shun Kurose, MD, ${ }^{2}$ Koichi Morisaki, MD, PhD, ${ }^{2}$ Kaku Nakano, PhD, ${ }^{3}$ \\ Jun-ichiro Koga, MD, PhD, ${ }^{3,4}$ Tadashi Furuyama, MD, PhD, ${ }^{2}$ Masaki Mori, MD, PhD, FACS, ${ }^{2}$ \\ and Kensuke Egashira, MD, PhD, FAHA, FESC 3,5
}

Two decades have passed since therapeutic angiogenesis was proposed to promote reparative collateral growth as an alternative therapy for ischemic diseases in patients for whom neither surgical revascularization nor endovascular therapy was suitable. When therapeutic angiogenesis first began, local administration was conducted using recombinant growth factor proteins or gene-encoding growth factors for endothelial cells. Since then, autologous stem cells and endothelial progenitor cell transplantation therapy

${ }^{1}$ Department of Vascular Surgery, Graduate School of Medical Sciences, International University of Health and Welfare, Narita, Chiba, Japan

${ }^{2}$ Department of Surgery and Science, Graduate School of Medical Sciences, Kyushu University, Fukuoka, Fukuoka, Japan

${ }^{3}$ Department of Cardiovascular Research, Development, and Translational Medicine, Center for Disruptive Cardiovascular Innovation, Kyushu University, Fukuoka, Fukuoka, Japan

${ }^{4}$ Department of Cardiovascular Medicine, Kyusyu University Graduate School of Medical Sciences, Fukuoka, Fukuoka, Japan

${ }^{5}$ Department of Translational Medicine, Kyushu University Graduate School of Pharmaceutical Sciences, Fukuoka, Fukuoka, Japan

Received: November 24, 2019; Accepted: November 25, 2019 Corresponding author: Takuya Matsumoto, MD, PhD, FACS. Department of Vascular Surgery, Graduate School of Medical Sciences, International University of Health and Welfare, 4-3 Kouzunomori, Narita, Chiba 286-8686, Japan

Tel: +81-476-20-7701, Fax: +81-476-20-7702

E-mail: tak@iuhw.ac.jp

(cc) BY-NC-SA (C2020 The Editorial Committee of Annals of Vascular Diseases. This article is distributed under the terms of the Creative Commons Attribution License, which permits use, distribution, and reproduction in any medium, provided the credit of the original work, a link to the license, and indication of any change are properly given, and the original work is not used for commercial purposes. Remixed or transformed contributions must be distributed under the same license as the original. have been developed. Although many clinical trials have been performed on patients, most therapies have not yet become standard treatments. We have developed a nanoparticle (NP)-mediated, drug-targeting delivery system using bioabsorbable poly-lactic/glycolic acid (PLGA) NPs. In several animal models, pitavastatin-incorporated (Pitava)NPs showed significant therapeutic effects on critical limb ischemia. Because PLGA NPs are delivered selectively to vascular endothelial cells after intramuscular administration, it is suggested that therapeutic angiogenesis/arteriogenesis plays an important role in the mechanism by which PitavaNPs exert beneficial therapeutic effects. To translate this to clinical medicine, we have performed studies and produced Pitava-NPs in compliance with good laboratory practice/ good manufacturing practice regulations, and completed a phase I/II clinical trial, reporting the safety and efficacy of Pitava-NP intramuscular injection for patients with critical limb ischemia. This review will focus on therapeutic angiogenesis/arteriogenesis for peripheral arterial disease induced by Pitava-NPs.

Keywords: drug delivery system, angiogenesis, arteriogenesis, peripheral arterial disease, statin

\section{Background and Purpose}

Peripheral arterial disease (PAD) is a common disorder that causes claudication, ischemic pain, skin ulceration, and gangrene in the lower extremities, and often leads to amputation. To rescue the affected limb, re-vascularization procedures such as surgical bypass and endovascular therapy are always necessary to address critical limb ischemia (CLI). However, the prognosis of CLI remains poor, and new forms of treatment to counteract the disease are an urgent necessity. We have been developing a novel nanotechnology-based drug delivery system (nanoDDS) to induce therapeutic angiogenesis/arteriogenesis by delivering statins, $\mathrm{HMG}-\mathrm{CoA}$ reductase inhibitors, to 
the endothelium. This review will summarize the current development status of this nano-DDS, including animal experiments and a phase I/IIa clinical study (Clinical Trial Registration Number: UMIN000008011).

\section{PAD}

PAD causes limb function disability (intermittent claudication [IC]) as well as resting pain or gangrene (CLI) by impairing the blood supply to peripheral tissues. With regard to the limb, PAD has a favorable prognosis because the claudication remains stable over a 10 -year period in $70 \%-80 \%$ of patients. ${ }^{1}$ Initially, conservative treatments such as medical management and/or exercise therapy should be recommended for IC. If therapeutic angiogenesis can improve the walking performance more effectively than the first-line drug cilostazol, $\left.{ }^{2}\right)$ therapeutic angiogenesis may become the standard therapy for IC.

CLI generally requires revascularization to improve the blood flow as soon as possible because untreated CLI ultimately leads to limb loss due to inadequate blood flow and oxygenation to the distal extremities. Even with the current standard therapies for CLI such as surgical bypass or endovascular treatment, the 5- and 10-year survival rates of patients are $50 \%$ and $10 \%$, respectively. ${ }^{3)}$ Despite its higher periprocedural morbidity and mortality, bypass surgery has been the gold standard treatment for patients with CLI because of the high volume of below-the-knee lesions associated with PAD. However, the patency rates after surgical bypass or endovascular treatment remain poor because of late intimal hyperplasia caused by poor distal runoff arteries. Therefore, to increase the patency rate of a bypass graft, it is necessary to improve the distal runoff vessels by therapeutic angiogenesis. In patients with such poor general condition, less invasive therapies like therapeutic angiogenesis are urgently needed.

\section{Poly (Lactic-co-Glycolic Acid) (PLGA) Nanoparticles (NPs)}

Prolonged and efficient expression of angiogenic factors has been the central strategy for therapeutic angiogenesis. For this purpose, delivery systems need to overcome physiological barriers, such as those between tissues and cells, to allow sufficient expression of angiogenic factors and decrease any potential toxicity that may lower the safe dose of the drug.

Compared with natural polymers, PLGA is an effective polymer for creation of $\mathrm{NPs}^{4}$ ) to encapsulate biologically active compounds or genes to sustain and control the release of the encapsulated DNA or drug over a period of several days to several weeks in a delivery system (nanoDDS). Specifically, PLGA is a safe, Good Manufacturing
Practice-graded, Food and Drug Agency-approved, biocompatible, and biodegradable polymer that can be used in clinical settings. PLGA NPs can improve the high therapeutic efficacy of gene or drug delivery into cells. PLGA itself has several interesting properties such as controlled and sustained release, ${ }^{4,5)}$ low cytotoxicity, long-standing biomedical applications, biocompatibility with tissues and cells, prolonged residence time, and targeted delivery.

\section{Analysis of Fluorescein Isothiocyanate (FITC)-NPs in a Mouse Model of Hindlimb Ischemia}

At $3,7,14$, and 21 days after intramuscular injection of FITC-NPs into a mouse model of hindlimb ischemia, the gastrocnemius muscle was isolated from ischemic and nonischemic limbs and examined under a fluorescence microscope.6) FITC fluorescence was strongest at day 3 in FITC-NP-injected ischemic hindlimb muscles and was detectable up to 14 days in these ischemic muscles only (Fig. 1A). FITC fluorescence was undetectable at day 3 in FITC-only-injected hindlimb muscles. On day 14, FITC signals were predominantly observed in capillaries and arterioles (Fig. 1B). In the FITC-NP-injected hindlimbs, immunofluorescence staining revealed that the FITC signals were mainly localized in CD31-positive endothelial cells (Fig. 1C).

\section{Statins}

Statins are widely used in the clinic for lipid-lowering purposes. Consumption is safe and well-tolerated by humans. Other than angiogenic effects, side effects of statins, such as development of atherosclerosis ${ }^{7}$ ) and cancer, ${ }^{8)}$ have not been recognized. Independent of their cholesterollowering effects, ${ }^{9}{ }^{1}$ statins also have pleiotropic effects. Several studies have reported that systemic administration of statins promotes re-endothelialization of damaged blood vessels and induces angiogenesis in ischemic tissues because statins target endothelial cells through their vasculoprotective effects and increase endothelial progenitor cells (EPCs) in the peripheral blood. ${ }^{10,11)}$

Several different statins are available for clinical use. Pitavastatin is minimally metabolized by CYP2C9, unlike other statins, including atorvastatin, fluvastatin, pravastatin, rosuvastatin, and simvastatin, that are mainly metabolized by CYP3A4. ${ }^{12)}$ In human umbilical vein endothelial cell (HUVEC) scratch motility assays, only pitavastatin increased the re-endothelialization response following scratch injury ${ }^{13)}$ (Fig. 2A). We investigated effects of statins and pitavastatin-NPs on the angiogenic capacity of HUVECs in vitro. Treatment with pitavastatin increased angiogenic activity in human endothelial cells, while other 


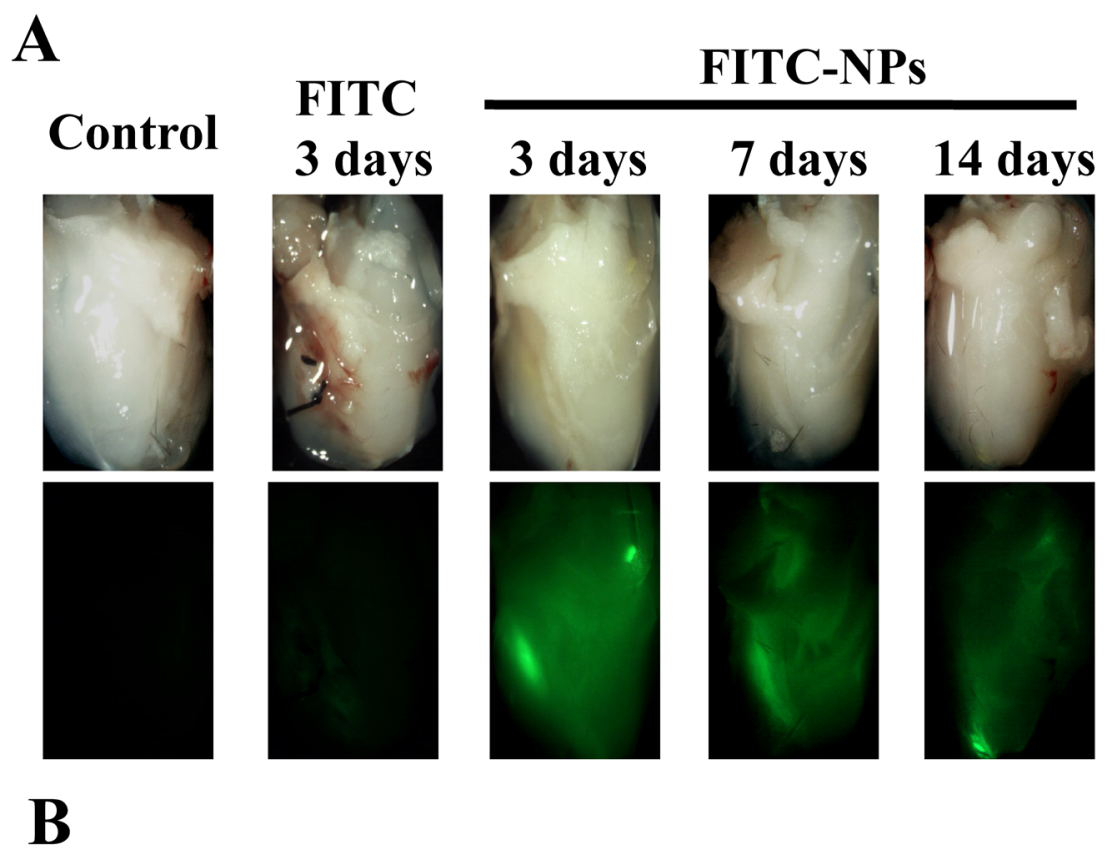

Control

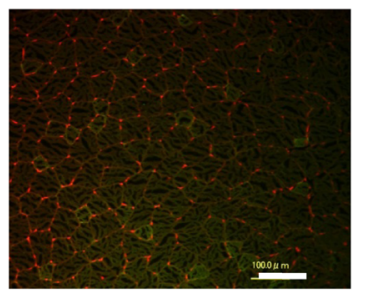

C

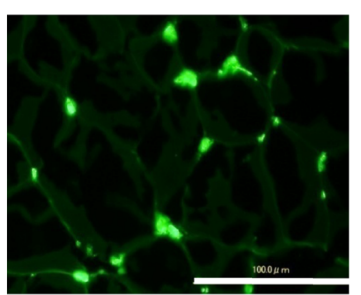

FITC-NPs

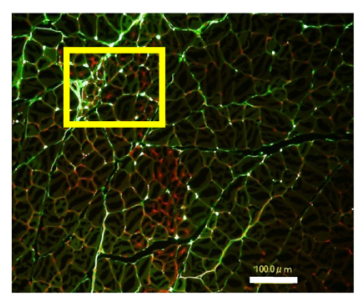

CD31

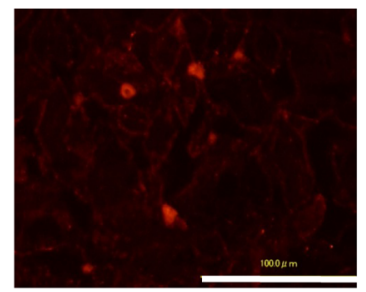

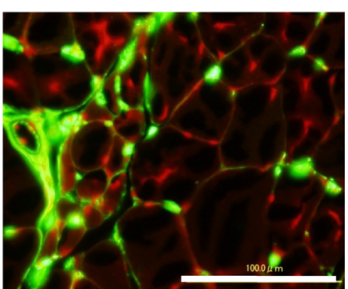

Merged

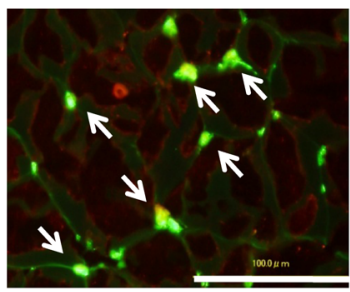

Fig. 1 (A) Representative light (upper panels) and fluorescence (lower panels) stereomicrographs of gastrocnemius muscles from nonischemic (control) and ischemic (FITC, FITC-NPs) hindlimbs. (B) Fluorescence micrographs of gastrocnemius muscle crosssections from non-ischemic mice with no injection (control) and ischemic mice at 14 days after injection of FITC-NPs (middle). An expanded view of the boxed area in the middle panel is shown in the right panel. Scale bars: $100 \mu \mathrm{m}$. (C). Immunofluorescence staining of ischemic muscles at 14 days after injection of FITC-NPs, shown as FITC (left panel), CD31 (endothelial cell detection; middle panel), and merged (right panel) images. FITC: fluorescein isothiocyanate; NPs: nanoparticles

statins had no apparent effect ${ }^{14)}$ (Fig. 2B). Treatment with Pitava-NPs also increased the angiogenic activity in HUVECs. The angiogenic activity of HUVECs was increased by $10 \mathrm{nM}$ Pitava-NPs, but equivalent dose of pitavastatin solution had no significant effects ${ }^{6}$ (Fig. 2C).

Oral daily administration of pitavastatin at $0.4 \mathrm{mg} / \mathrm{kg}$ for 14 days did not increase blood flow recovery, while pitavastatin at 1.0 and $10 \mathrm{mg} / \mathrm{kg}$ significantly increased blood flow recovery on day 14 . Single intramuscular injection of nanoparticulated soluble pitavastatin at $0.4 \mathrm{mg} / \mathrm{kg}$ significantly improved blood perfusion at 14 days after induction of hindlimb ischemia in rabbits ${ }^{14)}$ (Fig. 3A). Treatment with Pitava-NPs containing pitavastatin at $0.4 \mathrm{mg} / \mathrm{kg}$, but not with FITC-NPs or statin alone, sig- 
A
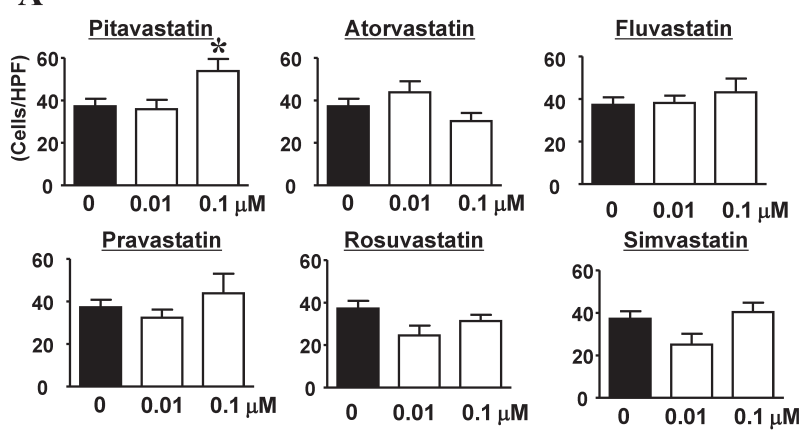

B

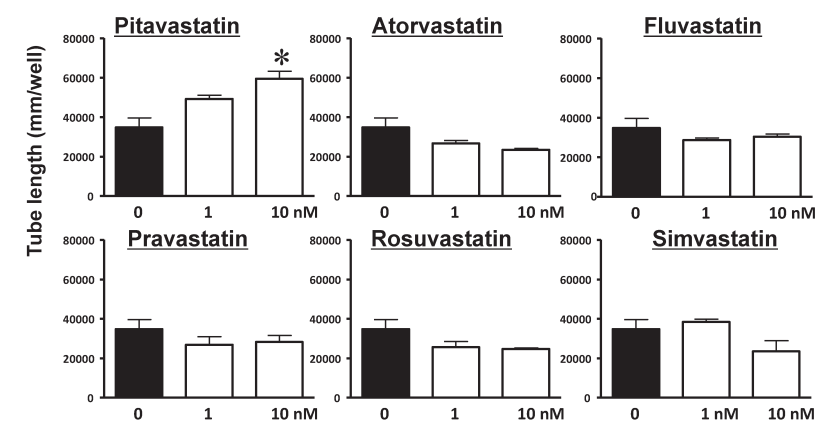

C

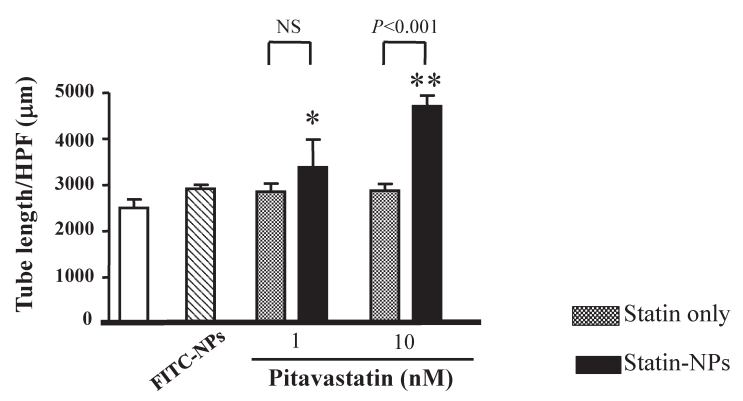

Fig. 2 Effects of pitavastatin on angiogenic capacity in vitro.

(A) Only pitavastatin promoted the re-endothelialization response in HUVEC scratch motility assays. The vertical axis denotes the number of migrated cells into the scratched area. ${ }^{*} \mathrm{P}<0.05$, vs. no treatment. (B) Treatment with pitavastatin only increased angiogenic activity in human endothelial cells. The vertical axis denotes tube formation. ${ }^{*} \mathrm{P}<0.01$, vs. no treatment. (C) Effects of statinNPs on angiogenic capacity of HUVECs in vitro. Treatment with Pitava-NPs increased angiogenic activity. The vertical axis denotes tube formation. ${ }^{*} \mathrm{P}<0.01,{ }^{*} \mathrm{P}<0.001$, vs. control. HUVEC: human umbilical vein endothelial cell; NPs: nanoparticles

nificantly increased blood flow recovery on days 7 and 14 after induction of hindlimb ischemia in mice (Figs. 3B and 3C). Therefore, our nano-DDS seems to be effective at an approximately 300 times lower dose than the cumulative systemic dose. Pitava-NPs reduced the risks of significant creatine kinase elevation and rhabdomyolysis.
A
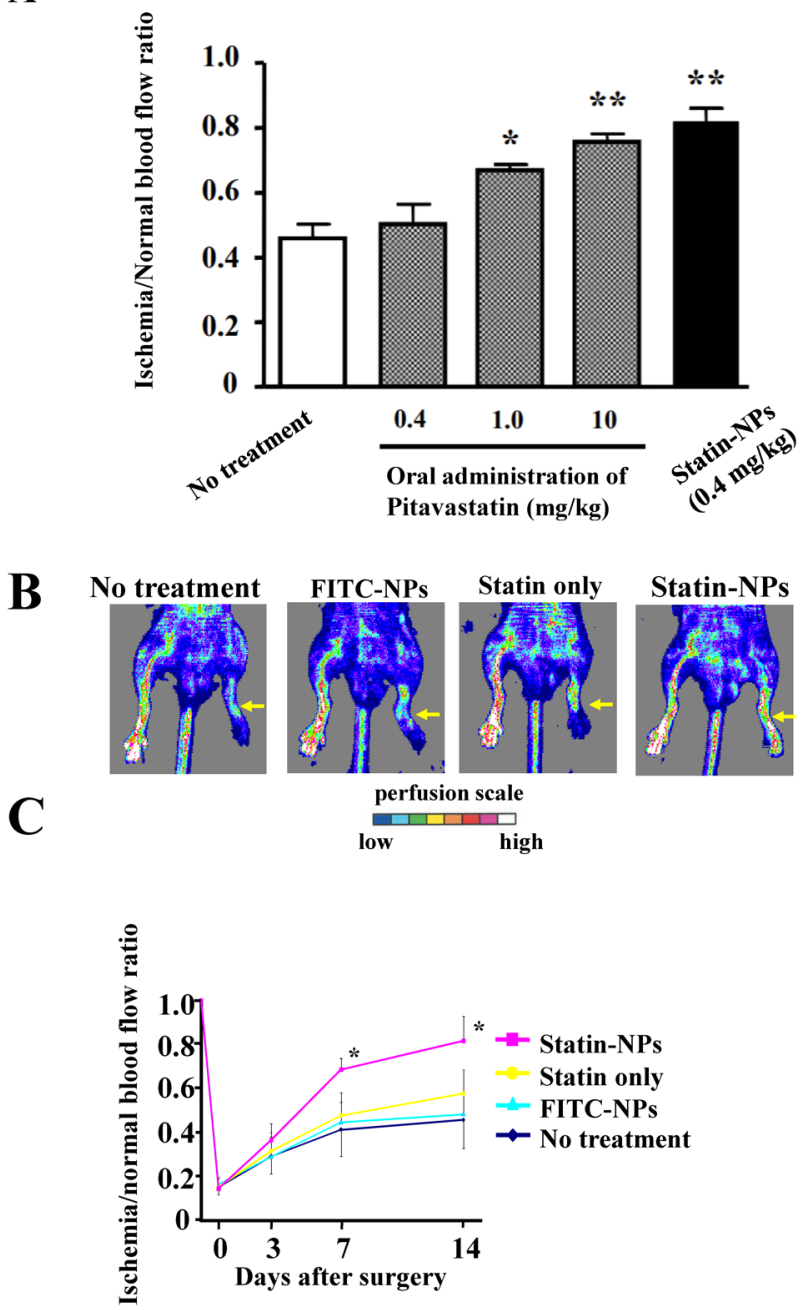

Fig. 3 (A) Effects of oral daily administration of pitavastatin on ischemia-induced neovascularization in rabbits. Laser Doppler perfusion imaging-derived blood flow recovery was quantified at 14 days. ${ }^{*} \mathrm{P}<0.05,{ }^{*} \mathrm{P}<0.01$, vs. no treatment group. (B) Representative laser Doppler perfusion images at 14 days post-ischemia in mice. The arrows indicate the ischemic limb. (C) Quantification of blood flow recovery in mice.

\section{Mechanisms of Pitavastatin-NP-Mediated Angiogenesis/Arteriogenesis}

Intramuscular injection of nanoparticulated soluble pitavastatin in mice significantly improved blood perfusion after induction of hindlimb ischemia without any effects on serum lipid levels, suggesting that Pitava-NPs exerted their efficacy by mechanisms independent from their lipid-lowering effects. $\left.{ }^{6}\right)$ However, both arteriogenesis and angiogenesis were significantly increased. Single intramuscular injection of non-nanoparticulated soluble pitavastatin at high dose compared with Pitava-NPs in mice had no effect on blood perfusion after induction of hindlimb ischemia. HMG-CoA reductase inhibitors mobi- 


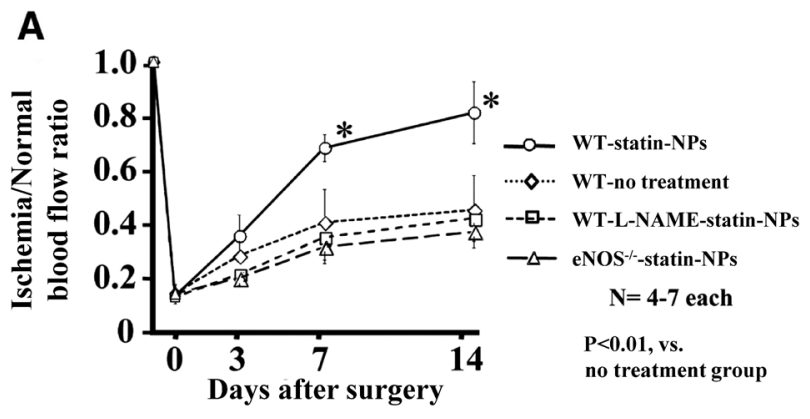

B
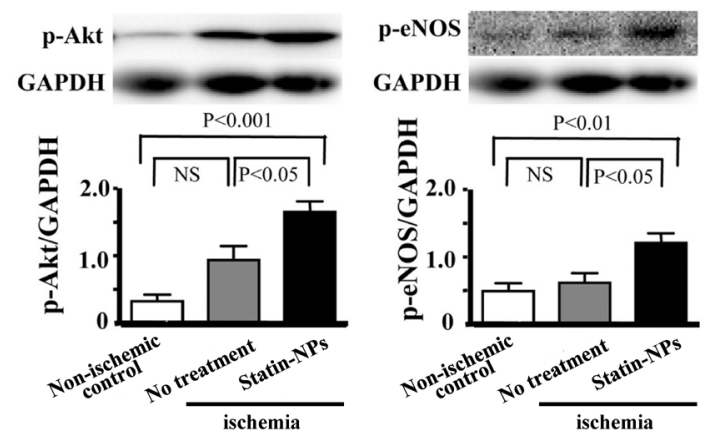

Fig. 4 (A) Quantification of blood flow recovery in wild-type (WT) mice with or without administration of $\mathrm{N} \omega$-nitro-L-arginine methyl ester hydrochloridean (L-NAME), a NOS inhibitor, and in eNOS ${ }^{-1-}$ mice. (B) western blot analysis of phosphorylated Akt and eNOS in ischemic and nonischemic muscles at 7 days after ischemia.

lized bone marrow-derived EPCs through stimulation of the Akt signaling pathway. ${ }^{15,16)}$ However, intramuscular injection of nanoparticulated soluble pitavastatin in mice did not increase the number of EPCs in the circulating blood.

No therapeutic effects of statin-NPs were observed in wild-type mice administered $\mathrm{N} \omega$-nitro-L-arginine methyl ester hydrochloridean (L-NAME) or in $\mathrm{eNOS}^{-1-}$ mice (Fig. 4), suggesting that eNOS-related signals are involved in the mechanism of statin-induced enhancement of ischemia-induced neovascularization. ${ }^{6)}$ Treatment with Pitava-NPs increased both phosphorylated eNOS and serine-threonine-specific protein kinase (Akt) in ischemic muscles compared with nonischemic control and nontreated ischemic muscles after 7 days of treatment. ${ }^{6)}$ Several studies demonstrated that Pitava-NPs activated the eNOS/PI3K/Akt pathway accompanied by induction of endogenous angiogenic factors like vascular endothelial growth factor and fibroblast growth factor-2. ${ }^{15,17)}$

\section{Proof of Functional Collateral Arteries in a Rabbit Model of Chronic Hindlimb Ischemia}

Before clinical approaches, studies on larger animals such as rabbits or cynomolgus monkeys are desirable to address the therapeutic effects of Pitava-NPs on the develop-
A
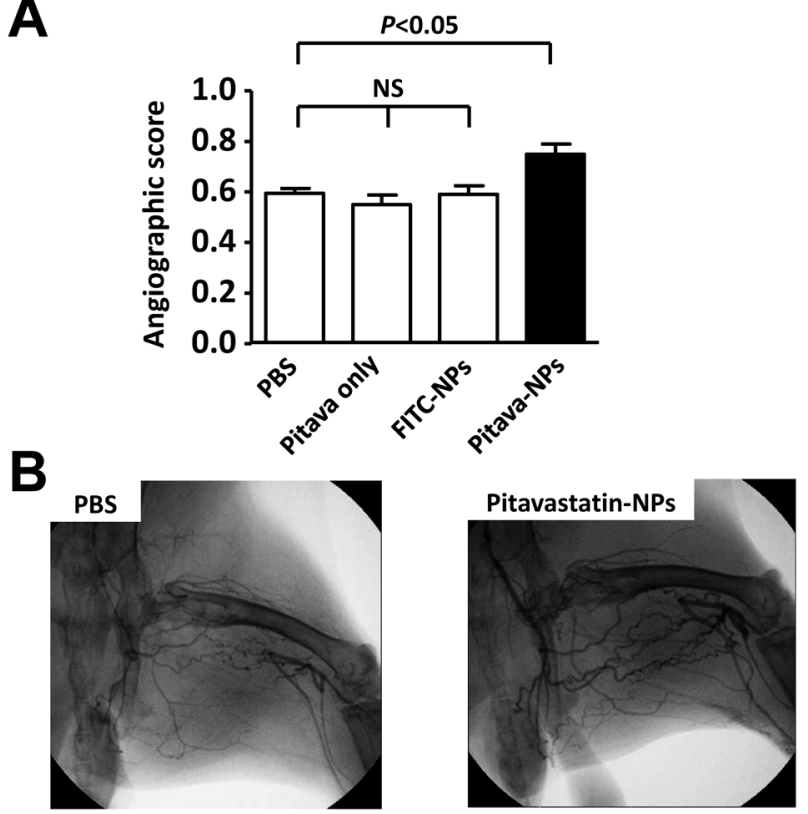

Fig. 5 Effects of Pitava-NPs on angiographically visible collateral arterial development.

(A) Quantitative data for the angiographic scores. (B) Representative angiograms of ischemic lower extremities in rabbits at 28 days after treatment. Corkscrew-like collateral arteries were only observed in the Pitava-NPs group. FITC: fluorescein isothiocyanate; NPs: nanoparticles; PBS: phosphate- buffered saline

ment of collateral arteries. Male Japanese White rabbits were used to confirm the therapeutic effects of Pitava-NPs on the development of collateral arteries and restoration of tissue perfusion in a model of chronic hindlimb ischemia. Angiography at 28 days after treatment showed that the Pitava-NPs group developed more corkscrew-like collateral arteries compared with the control and pitavastatin alone groups (Figs. 5A and 5B). ${ }^{14)}$ Furthermore, use of the nano-DDS and endothelial cell-selective delivery of pitavastatin increased the development of collateral arteries at an approximately 100 times lower dose than the cumulative systemic dose and improved exerciseinduced ischemia in a rabbit model of chronic hindlimb ischemia. ${ }^{14)}$

\section{Evaluation of Angio-Computed Tomogra- phy (CT) Imaging-Visible Collateral Arterial Development by Pitava-NPs in a Cynomol- gus Monkey Model of Chronic Hindlimb Ischemia}

To evaluate therapeutic angiogenesis/arteriogenesis in a nonhuman primate model for a preclinical proof-of-concept study, we used male cynomolgus monkeys weighing $3-4 \mathrm{~kg}$ and created chronic hind limb ischemia by surgical removal of the right femoral artery. There are three rea- 
sons why a nonhuman primate model is a beneficial test subject. First, this model of chronic hind limb ischemia had a more similar degree of ischemia to patients with PAD than the acute hind limb ischemia model in mice and rabbits. Second, the collateral arterial development in the cynomolgus monkey model was detected by threedimensional angio-CT, similar to the evaluation method used in clinical practice ${ }^{18)}$ (Fig. 6A). Third, the functional analysis of Pitava-NPs in the model was performed in a similar manner as in clinical patients.

One week after induction of chronic hind limb ischemia, the monkeys were injected intramuscularly at 10 injection sites in regions of the ischemic limb with saline (vehicle), FITC-NPs, pitavastatin (0.5 mg per body), and Pitava-NPs

\section{A}

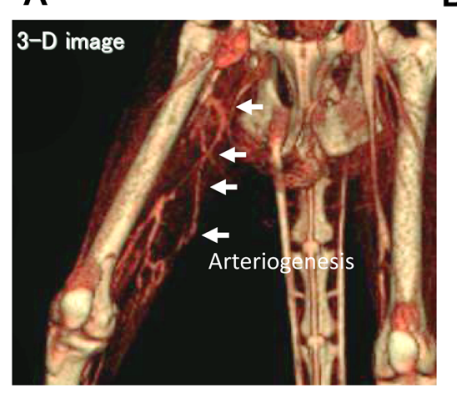

B

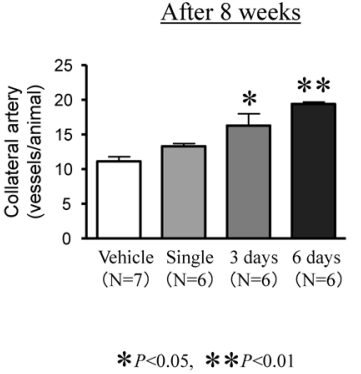

Fig. 6 (A) Angio-CT imaging detected collateral arterial development at 28 days after daily intramuscular injections of Pitava-NPs on a 6-day protocol in a nonhuman primate chronic hindlimb model. (B) Effects of Pitava-NPs on angio-CT imaging-visible collateral arterial development at 28 days after treatment in a nonhuman primate model. NPs: nanoparticles incorporated with $0.5 \mathrm{mg} /$ body of pitavastatin; animals were divided into single injection, 3-day daily injection, and 6-day daily injection groups. The three-dimensional angio-CT was performed to evaluate collateral arterial development after 8 weeks of treatment. Treatment with FITC-NPs or pitavastatin had no effects on the number of angio-CT-detectable collateral channels (data not shown). However, the 3- and 6-day daily treatments with PitavaNPs significantly increased the number of developed collateral arteries as compared with saline (Fig. 6B). Single injection of Pitava-NPs showed no significant effects (Fig. 6B).

\section{Phase I/lla, Open-Label, Dose-Escalation Study}

The results of the preclinical studies in mice, rabbits, and monkeys suggested that intramuscular treatment with Pitava-NPs at the clinically approved dose range had higher efficacy than pitavastatin alone. Intramuscular administration once daily at 20 injection sites for 5 days was considered a clinically feasible protocol.

Thus, after the approval of study design by Japanese regulatory agency, we performed a phase I/IIa, open-label, four-dose-escalation clinical study and reported the safety, tolerability, and therapeutic efficacy of five consecutive days of intramuscular administration of Pitava-NPs in 16 patients with PAD (Fig. 7).

\section{Study Design}

After a pretesting observation period for 30 days, intra-

\section{Study protocol}

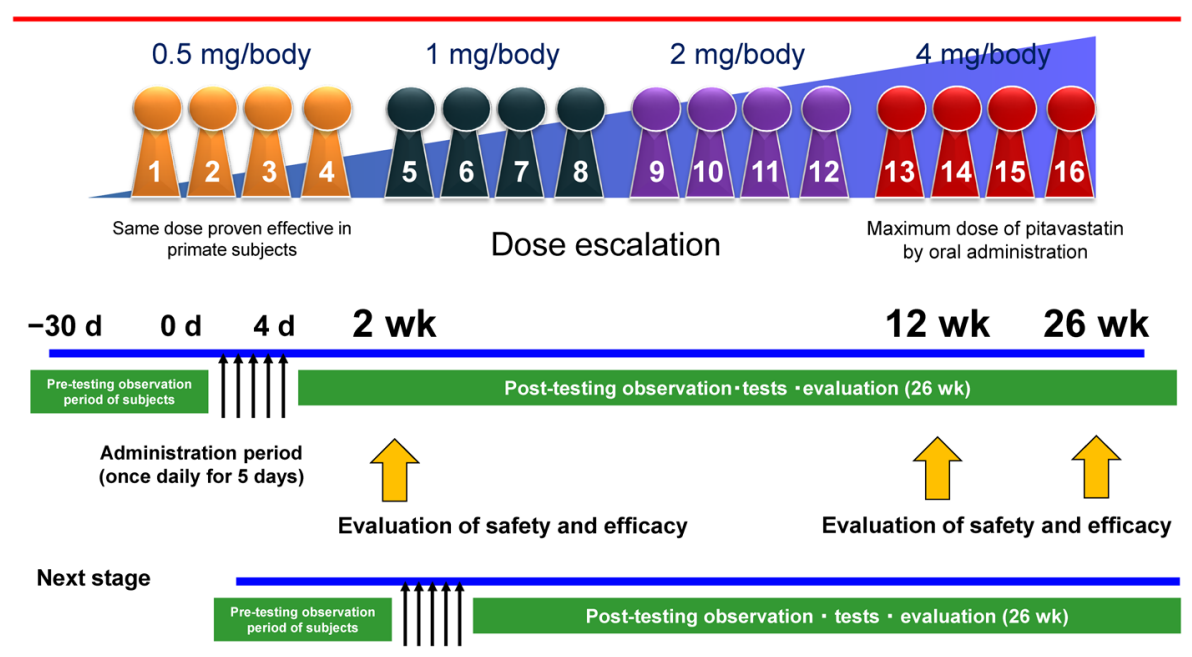

Dose escalation was approved after evaluation of safety and efficacy in the 4 subjects on a given dose

Fig. 7 Protocol of the phase I/lla, open-label, dose-escalation study. 
muscular administration of Pitava-NPs containing 0.5, 1 , 2 , or $4 \mathrm{mg}$ pitavastatin calcium at 20 sites for five consecutive days was performed in a single affected leg. The 20 injection sites in the leg were the same for each administration according to the procedure manual for Pitava-NPs administration. Patient follow-up to collect assessment data was performed monthly up to 26 weeks ( \pm 7 days at each visit) after the final administration of Pitava-NPs. At 2, 12, and 26 weeks after the final administration of Pitava-NPs, the safety, tolerability, and possible therapeutic efficacy were assessed. The pharmacokinetics and metabolites of the parent compound and the lactone bodies of pitavastatin were assessed at $0.5,1,2,4,8,12$, and $24 \mathrm{~h}$ after the first and final administration for plasma and before the first administration, and at 12 and $24 \mathrm{~h}$ after the first administration and 12 and $24 \mathrm{~h}$ after the final administration for urine. The principal investigator evaluated the safety and pharmacokinetic results by 2 weeks after the final administration of each dose and approved dose escalation (Fig. 7). The study design, protocol, and informed consent forms were approved by the institutional ethical committees and the Pharmaceutical and Medical Device Agency. The study was conducted, recorded, and reported in compliance with the principles of the Declaration of Helsinki and Guideline for Good Clinical Practice (Ordinance of the Ministry of Health, Labor and Welfare) under the support of the Clinical and Translational Research Center of Kyushu University Hospital. The trial was registered under Clinical Trial Registration Number UMIN000008011. All patients who satisfied the inclusion criteria provided written informed consent before enrollment in the study.

\section{Patient Cohort}

Patients were enrolled when they (i) had chronic CLI including resting pain or ischemic ulcer (Fontaine grade III or IV) that was resistant to standard medication for at least 2 weeks; (ii) were contraindicated for surgical or catheter revascularization based on usual practice standards; and (iii) were aged $\geq 20$ years at the time of providing consent. The patient eligibility criteria and possible ethical discussion points of the trial based on the Declaration of Helsinki were confirmed by the institutional review board at Kyushu University, and approved by the Ministry of Health, Labor and Welfare.

\section{Primary Endpoint: Safety and Efficacy}

The primary endpoint of the safety assessment was to confirm serious adverse events and side effects caused by Pitava-NPs, including the degrees and ratios of these events. The other two primary endpoints were to assess
(1) the pharmacokinetics of pitavastatin and its metabolite (pitavastatin lactone) in the plasma/urine, and (2) the efficacy of treatment, such as changes in Fontaine classification and Rutherford classification.

\section{Secondary Endpoints: Efficacy-Related Parameters}

The secondary endpoints were physiological tests (body weight, body temperature, blood pressure, pulse), clinical laboratory tests, cardiac function tests, and efficacy of treatment including ankle-brachial index in supine position, toe-brachial index in supine position, ankle pressure in supine position, pulse volume recording, laser Doppler blood flow, angiography, ulcer size, degree of pain, transcutaneous oxygen pressure, number of cases with minor amputation, and number of cases with major amputation.

\section{Pitava-NPs: Pitavastatin-Incorporating PLGA NPs}

Pitava-NPs were supplied by Kowa Company Ltd. (Nagoya, Japan) as a sterile powder including $1 \mathrm{mg}$ of pitavastatin calcium per vial. The total dose of NK-104-NPs was suspended, adjusted to a final volume of $20 \mathrm{~mL}$ with physiologic saline (Otsuka Pharmaceutical Factory, Inc., Tokyo, Japan), and administered within $1 \mathrm{~h}$. The $20 \mathrm{~mL}$ of physiologic saline was divided $20 \times$ into 1 - $\mathrm{mL}$ syringes, and the 1 - $\mathrm{mL}$ amount was administered intramuscularly at 20 sites on the lower limb once daily for 5 days. The 20 administration sites on the lower limb were determined in accordance with the separately prepared administration manual for the test product. The dose per site was approximately $1 \mathrm{~mL}$ and markings were made on the limb to ensure that the injection sites did not overlap.

\section{Data Collection and Statistical Analysis}

All data were collected and analyzed at the Data Center of the Center for Clinical and Translational Research at Kyushu University Hospital. Pharmacokinetics parameters were estimated at the Department of Clinical Pharmacokinetics. Subjects who were administered the test products and had available data were analyzed for safety, pharmacokinetics, and possible therapeutic efficacy. For background information, the basic statistics in each group and the whole cohort were calculated.

\section{Results}

Although several serious adverse events were observed, such as death or other critical events, none of them were drug-related. However, there were minor events that could 
have been caused by the drug. No dose-response relationship was observed.

The average of clinical laboratory data did not significantly change and remained in normal range before and after administration of Pitava-NPs.

Changes in the results of physiological tests and cardiac function tests at each point when compared with base line data were not observed. There were no important abnormalities reported as adverse events.

Although no significant change of the ankle-brachial pressure index and toe pressure index were observed, some patients administered with Pitava-NPs containing 2 and $4 \mathrm{mg}$ pitavastatin calcium improved in Fontaine classification and Rutherford classification.

Maximum serum concentration and area under the blood concentration-time curve (AUC) from 0 to $1 \mathrm{~h}$ of pitavastatin and its metabolite increased according to the dose-response of Pitava-NPs. After 5 days of administration, the AUC slightly but insignificantly increased at 5 days compared to the results of day- 1 administration.

\section{Conclusion}

We have summarized the efficacy and safety of Pitava-NPs in preclinical studies including animal models of hindlimb ischemia and in a phase I/IIa clinical study in patients with CLI.

The current applications of the nano-DDS for PAD mainly utilize the following three major mechanisms for drug delivery: highly efficient delivery of encapsulated drugs into the cellular cytoplasm, slow cytoplasmic drug release, and vascular endothelial cell-targeted delivery. These properties of the nano-DDS may be applicable for treatment of PAD, the concept of which was proven by the proof-of-concept study using Pitava-NPs in the hindlimb ischemia models in mice, rabbits, and monkeys. In addition to the therapeutic effects of the nano-DDS on endothelial cells after oral administration of the drug, there is a wide variety of ways to combine the nano-DDS and pitavastatin, which may expand the possibilities of the current pharmacotherapy for PAD. We have completed the phase I/IIa investigator-initiated clinical trial at $\mathrm{Ky}$ ushu University Hospital to test the safety and efficacy of NP-mediated delivery of pitavastatin in patients with CLI (UMIN000008011). The results of further clinical trials may prove the safety and efficacy of Pitava-NPs in CLI patients in the near future.

\section{Acknowledgments}

The described studies were supported by Grants-in-Aid for Scientific Research from the Ministry of Education, Science, and Culture, Tokyo, Japan, 25293185 (K. Egashi- ra), by Health Science Research Grants (Research on Translational Research, Intractable Diseases, and Nanomedicine) from the Ministry of Health Labor and Welfare, Tokyo, Japan (K. Egashira), and by the Intractable diseases overcome research project from Japan Agency for Medical Research and development (K. Egashira).

They were also partly supported by the Center for Clinical and Translational Research at Kyushu University Hospital.

The authors thank Alison Sherwin from Edanz Group (www.edanzediting.com/ac) for editing a draft of this manuscript.

\section{Disclosure Statement}

Dr. Egashira is the inventor of an issued patent on the results reported in the present study (Pharmaceutical composition containing statin-encapsulated nanoparticle, WO 2008/026702).

\section{Author Contributions}

Study conception: TM, KE, MM

Data collection: SY, SK, KM, KN

Analysis: SY, KN

Investigation: TM, KE, TF

Writing: TM, KE, JK

Critical review and revision: all authors

Final approval of the article: all authors

Accountability for all aspects of the work: all authors

\section{References}

1) Hirsch AT, Haskal ZJ, Hertzer NR, et al. ACC/AHA 2005 guidelines for the management of patients with peripheral arterial disease (lower extremity, renal, mesenteric, and abdominal aortic): executive summary a collaborative report from the American Association for Vascular Surgery/ Society for Vascular Surgery, Society for Cardiovascular Angiography and Interventions, Society for Vascular Medicine and Biology, Society of Interventional Radiology, and the ACC/AHA Task Force on Practice Guidelines (Writing Committee to Develop Guidelines for the Management of Patients With Peripheral Arterial Disease): endorsed by the American Association of Cardiovascular and Pulmonary Rehabilitation; National Heart, Lung, and Blood Institute; Society for Vascular Nursing; TransAtlantic Inter-Society Consensus; and Vascular Disease Foundation. J Am Coll Cardiol 2006; 47: 1239-312.

2) Regensteiner JG, Ware JE Jr, McCarthy WJ, et al. Effect of cilostazol on treadmill walking, community-based walking ability, and health-related quality of life in patients with intermittent claudication due to peripheral arterial disease: meta-analysis of six randomized controlled trials. J Am Geriatr Soc 2002; 50: 1939-46.

3) Norgren L, Hiatt WR, Dormandy JA, et al. Inter-society 
consensus for the management of peripheral arterial disease (TASC II). J Vasc Surg 2007; 45 Suppl S: S5-67.

4) Kawashima Y, Yamamoto $H$, Takeuchi H, et al. Properties of a peptide containing DL-lactide/glycolide copolymer nanospheres prepared by novel emulsion solvent diffusion methods. Eur J Pharm Biopharm 1998; 45: 41-8.

5) Panyam J, Zhou W-Z, Prabha S, et al. Rapid endo-lysosomal escape of poly (DL-lactide-co-glycolide) nanoparticles: implications for drug and gene delivery. FASEB J 2002; 16 : 1217-26.

6) Kubo M, Egashira K, Inoue T, et al. Therapeutic neovascularization by nanotechnology-mediated cell-selective delivery of pitavastatin into the vascular endothelium. Arterioscler Thromb Vasc Biol 2009; 29: 796-801.

7) Kitamoto S, Nakano K, Hirouchi Y, et al. Cholesterollowering independent regression and stabilization of atherosclerotic lesions by pravastatin and by antimonocyte chemoattractant protein-1 therapy in nonhuman primates. Arterioscler Thromb Vasc Biol 2004; 24: 1522-8.

8) Sata M, Nishimatsu H, Osuga J, et al. Statins augment collateral growth in response to ischemia but they do not promote cancer and atherosclerosis. Hypertension 2004; 43: 121420.

9) Takemoto M, Liao JK. Pleiotropic effects of 3-hydroxy-3methylglutaryl coenzyme a reductase inhibitors. Arterioscler Thromb Vasc Biol 2001; 21: 1712-9.

10) Sata M, Nishimatsu H, Suzuki E, et al. Endothelial nitric oxide synthase is essential for the HMG-CoA reductase inhibitor cerivastatin to promote collateral growth in response to ischemia. FASEB J 2001; 15: 2530-2.

11) Kureishi Y, Luo Z, Shiojima I, et al. The HMG-CoA reductase inhibitor simvastatin activates the protein kinase Akt and promotes angiogenesis in normocholesterolemic animals. Nat Med 2000; 6: 1004-10.

12) Sweetman SC. Cardiovascular drugs. In: Sweetman SC ed. Martindale: The Complete Drug Reference (36th edition). London: Pharmaceutical Press, 2009: 1155-434.

13) Tsukie N, Nakano K, Matoba T, et al. Pitavastatinincorporated nanoparticle-eluting stents attenuate in-stent stenosis without delayed endothelial healing effects in a porcine coronary artery model. J Atheroscler Thromb 2013; 20: 32-45.

14) Oda S, Nagahama R, Nakano K, et al. Nanoparticlemediated endothelial cell-selective delivery of pitavastatin induces functional collateral arteries (therapeutic arteriogenesis) in a rabbit model of chronic hind limb ischemia. J Vasc Surg 2010; 52: 412-20.

15) Llevadot J, Murasawa S, Kureishi Y, et al. HMG-CoA reductase inhibitor mobilizes bone marrow-derived endothelial progenitor cells. J Clin Invest 2001; 108: 399-405.

16) Altieri DC. Statins' benefits begin to sprout. J Clin Invest 2001; 108: 365-6.

17) Dimmeler S, Aicher A, Vasa M, et al. HMG-CoA reductase inhibitors (statins) increase endothelial progenitor cells via the PI 3-kinase/Akt pathway. J Clin Invest 2001; 108: 391-7.

18) Nakano K, Koga J, Egashira K. Nanoparticle-mediated endothelial cell-selective drug delivery system. Therapeutic Angiogenesis. 2017; 247-66. 